\title{
CLASSIFICAÇÃO E CRESCIMENTO DE UNIDADES DE VEGETAÇÃO EM FLORESTA OMBRÓFILA MISTA, SÃO FRANCISCO DE PAULA, RS ${ }^{1}$ \\ CLASSIFICATION AND GROWTH OF VEGETATION UNITS IN MIXED RAINY FOREST, SÃO FRANCISCO DE PAULA, RS
}

\author{
Juliana Fernandes Gomes ${ }^{2}$ Solon Jonas Longhi ${ }^{3} \quad$ Maristela Machado Araújo $^{4}$ Doádi Antônio Brena ${ }^{4}$
}

\section{RESUMO}

Este trabalho foi desenvolvido com o objetivo estudar diferentes grupos ecológicos presentes na Floresta Ombrófila Mista da FLONA de São Francisco de Paula, RS, e descrever sua composição e dinâmica de crescimento nas quatro estações do ano. Na avaliação, foram utilizados dez conglomerados permanentes de 1 ha cada $(100 \times 100 \mathrm{~m})$, os quais foram divididos em dez faixas de $10 \times 100 \mathrm{~m}\left(1000 \mathrm{~m}^{2}\right)$ e estas subdivididas em dez subunidades de $10 \times 10 \mathrm{~m}\left(100 \mathrm{~m}^{2}\right)$. Para a pesquisa, foi sorteada uma faixa de $10 \mathrm{x}$ $100 \mathrm{~m}$ em cada conglomerado, totalizando cem unidades amostrais em que foram instaladas, na altura do DAP, bandas dendrométricas em todas as árvores com CAP $\geq 30 \mathrm{~cm}$, para acompanhar o crescimento anual e nas quatro estações do ano. Os dados de densidade por espécie formaram uma matriz (parcela x espécie), que foi utilizada na análise de agrupamento da vegetação pelo do método de TWINSPAN (Two-way Indicador Species Analisys) na qual foi constatada a presença de três grupos ecológicos. O grupo 1 foi identificado como floresta primária, o grupo 2 floresta de locais úmidos e o grupo 3 floresta secundária. $\mathrm{O}$ incremento médio anual foi maior para floresta primária, seguido por floresta secundária e floresta de locais úmidos. Em ordem decrescente, o incremento médio periódico foi maior no verão, primavera, outono e inverno. As espécies que se destacaram em termos de densidade e incremento foram Cupania vernalis e Nectandra megapotamica.

Palavras-chave: Floresta Nativa; análise multivariada; TWINSPAN; incremento em diâmetro.

\section{ABSTRACT}

This work was developed with the objective of studying the present ecological groups in the Mixed Rainy Forest and of describing the composition and the growth dynamics during the four seasons. The work had been accomplished in ten permanent conglomerates of 1 ha $(100 \mathrm{~m} \times 100 \mathrm{~m})$, which were divided into ten strips of $10 \mathrm{~m} \times 100 \mathrm{~m}\left(1000 \mathrm{~m}^{2}\right)$ and these ones subdivided into ten subunits of $10 \mathrm{~m} \times 10 \mathrm{~m}\left(100 \mathrm{~m}^{2}\right)$. In this research, one $10 \mathrm{~m} \times 100 \mathrm{~m}$ strip in conglomerate was selected, totaling ten subunits, where it had been installed, at BHD height, dendrometric bands in all trees with $\mathrm{CBH} \geq 30 \mathrm{~cm}$, to verify the annual and the seasonal growth. The density per species formed a matrix $(99 \times 39)$ used to test vegetation grouping through TWINSPAN (Two-way indicator species analysis), since three ecological groups were verified. Group 1 was denominated primary forest; group 2, humid site forests, and group 3, secondary forest. $\geq 30 \mathrm{~cm}$. The annual average increment was larger for the primary forest, following the secondary forest and the forest of humid places. In decreasing order, the average periodic increment was larger in the summer, spring, autumn and winter. The species that stood out in density terms and increment were Cupania vernalis and Nectandra megapotamica.

Keywords: native Forest; multivariate analysis; TWINSPAN; increment in diameter.

\section{INTRODUÇÃO}

No estado do Rio Grande do Sul, ocorrem tipologias florestais distintas, diferenciadas por suas

1. Parte da dissertação de mestrado da autora apresentada no Programa de Pós-Graduação em Engenharia Florestal da Universidade Federal de Santa Maria.

2. Engenheira Florestal, Mestre pelo Programa de Pós-graduação em Engenharia Florestal, Centro de Ciências Rurais, Universidade Federal de Santa Maria, Av. Roraima, 1000, CEP 97105-900, Santa Maria (RS). julianafgomes@terra.com.br

3. Engenheiro Florestal, Dr., Professor Titular, Departamento de Ciências Florestais, Centro de Ciências Rurais, Universidade Federal de Santa Maria, Av. Roraima, 1000, CEP 97105-900, Santa Maria, RS. longhi.solon@gmail.com

4. Engenheiro(a) Florestal, Dr(a)., Professor(a) Adjunto(a), Departamento de Ciências Florestais, Centro de Ciências Rurais, Universidade Federal de Santa Maria, Av. Roraima, 1000, CEP 97105-900, Santa Maria, RS. araujo.maristela@gmail.com/dabrena@via-rs.net

Recebido para publicação em 8/03/2007 e aceito em 11/12/2007. 
composição florística e estrutura, entre as quais ocorre a Floresta Ombrófila Mista. Essa tipologia é reconhecida como um ecossistema de elevada riqueza em que, associado às espécies de Angiospermas, ocorre o predomínio da Gimnosperma, Araucaria angustifolia (Bertol.) Kuntze (Pinheiro-brasileiro).

Conforme Lamprecht (1990), as florestas nativas de araucária, que outrora se distribuíam em $250.000 \mathrm{Km}^{2}$, foram praticamente exterminadas, restando $0,7 \%$ no estado original e $6 \%$ como florestas secundárias.

A distribuição natural dos pinheirais no Rio Grande do Sul está relacionada com a irregularidade do terreno, considerando que o agrupamento da espécie é mais expressivo, sobretudo nos vales, nas encostas superiores dos rios e nos terrenos acidentados dos campos do planalto central e oriental (REITZ e KLEIN, 1966).

Conforme Gauto (1997), o crescimento de uma floresta ou das árvores presentes nesta, representa as mudanças ocorridas durante um determinado período de tempo. $\mathrm{O}$ desenvolvimento radial ou diamétrico varia expressivamente entre e dentro das espécies arbóreas, de acordo com a idade, estações do ano e condições microclimáticas (HIGUSHI et al., 2003).

O manejo das florestas nativas depende do conhecimento dos processos de dinâmica de seu crescimento, como também das técnicas de intervenções silviculturais que afetarão o crescimento das árvores (SOUZA et al., 1993).

A avaliação contínua dos incrementos em circunferência do tronco das espécies arbóreas possibilitará, a médio ou longo prazo, a determinação do ritmo e da taxa de crescimento, da periodicidade da atividade cambial e da influência dos fatores climáticos. Conforme Botosso e Tomazello Filho (2001), dentre as técnicas disponíveis para o monitoramento do crescimento do tronco das árvores, destacam-se as bandas (cintas) dendrométricas, pela facilidade de montagem, instalação, manutenção em condições de campo, execução da leitura e precisão, além do baixo custo.

O agrupamento de amostras com características bióticas e ou abióticas similares é um método de se analisar as comunidades, utilizando-se variáveis de acordo com o objetivo do trabalho. Esse procedimento permite descrever a estrutura, composição e a extensão das suas unidades funcionais de forma mais sintética e clara. A necessidade do conhecimento dos padrões sucessionais de uma vegetação é um exemplo claro da tendência de simplificar o entendimento dos ecossistemas (SANTOS et al., 2004).

A análise multivariada permite definir padrões nos dados. As três estratégias básicas de análise multivariada são: direta, por gradiente; ordenação; e classificação. As técnicas de ordenação e classificação organizam dados de comunidades baseadas em variáveis como a abundância de espécies, independentemente dos dados ambientais, sendo a interpretação ambiental uma etapa consecutiva (FELFILI et al., 2001).

O Twinspan (Two Way Indicator Species Analysis) é um método de classificação que permite agrupamento em estudos de vegetação. Este verifica a ocorrência de padrões na distribuição de espécies, em função às condições ambientais do local constatadas no campo, espécies indicadoras e preferenciais. $\mathrm{O}$ método é divisivo, hierárquico, politético, desenvolvido e discutido em um contexto fitossociológico em que a matriz de dados é construída pela abundância das espécies nas parcelas, e a partir da aplicação do método são formados agrupamentos por sucessivas divisões (HILL, 1979).

A cada divisão gera um autovalor que representa a variação dos dados no eixo de ordenação (MCCUNE e MEFFORD, 1997). Conforme Gauch (1982) os autovalores acima de 0,30 explicam a variação encontrada em dados obtidos na natureza.

O resultado gerado pelo método consiste numa tabela dicotômica, a qual indica o agrupamento das espécies e das unidades amostrais (FELFILI et al., 2001).

Considerando o número reduzido de estudos que abordam os fatores que influenciam no crescimento em Floresta Ombrófila Mista no Rio Grande do Sul, em paradoxo à importância da conservação e o uso apropriado de florestas naturais, torna-se necessário entender a dinâmica do ecossistema, a fim de subsidiar informações para o manejo sustentável.

Nesse contexto, este trabalho teve como objetivo identificar os agrupamentos na Floresta Nacional de São Francisco de Paula e caracterizar o incremento da vegetação e espécies predominantes nos grupos, 
nas quatro estações do ano.

\section{MATERIAL E MÉTODOS}

\section{Área de Estudo}

O presente estudo foi realizado na Floresta Nacional de São Francisco de Paula, administrada pelo IBAMA (Instituto Brasileiro do Meio Ambiente). A área de estudo está localizada entre as coordenadas $29^{\circ} 23^{\prime}$ e $29^{\circ} 27^{\prime} \mathrm{S}, 50^{\circ} 23^{\prime}$ e $50^{\circ} 25^{\prime} \mathrm{O}$, no Rincão dos Kröeff, distante $27 \mathrm{Km}$ município de São Francisco de Paula, RS.

O clima da região, de acordo com a classificação de Köppen, é do tipo "Cfb", mesotérmico médio (MORENO, 1961), o qual domina nas cotas altimétricas entre 1.000 e $1.100 \mathrm{~m}$ no norte do Rio Grande do Sul. Segundo Nimer (1990) nessa região, pelo menos um mês no ano apresenta temperatura permanece inferior a $10^{\circ} \mathrm{C}$, caracterizando inverno intenso cujo frio é constante durante o dia e a noite, e o calor típico do verão é praticamente ausente, considerando o efeito da altitude.

A vegetação da área de estudo pertence a uma zona de transição entre a Floresta Ombrófila Densa (Mata Atlântica) e a Floresta Ombrófila Mista, possuindo, assim, espécies endêmicas.

A mata em questão tem como característica o predomínio da Araucaria angustifolia. A Mata Atlântica, que faz parte da transição, ocorre nos vales dos cânions da área, contribuindo com algumas espécies que dispersam para a Mata de Araucária.

Métodos de coleta dos dados

O estudo foi realizado na área de pesquisa do Projeto Ecológico de Longa Duração (PELD), tomando-se como base dez unidades amostrais de 1 ha cada, divididas em dez faixas de $10 \times 100 \mathrm{~m}(0,1 \mathrm{ha})$, que por sua vez foram subdivididas em cem subparcelas de $10 \times 10 \mathrm{~m}$. Em cada unidade amostral, foi selecionada sistematicamente uma faixa onde foram avaliados os indivíduos com circunferência a altura do peito (CAP) igual ou maior de $30 \mathrm{~cm}$. Foram avaliados os parâmetros biométricos (CAP, altura comercial e total) e florístico. As faixas foram divididas em subparcelas de $10 \times 10 \mathrm{~m}$. Os indivíduos amostrados foram identificados, coletando-se material botânico para ser herborizado no Herbário do Departamento de Ciências Florestais (HDCF), da Universidade Federal de Santa Maria.

A avaliação do crescimento foi realizada partindo de bandas (cintas) dendrométricas instaladas em todos os indivíduos com CAP $\geq 30 \mathrm{~cm}$ presentes nas cem subparcelas. As medições foram realizadas durante 2 anos (2002 a 2004) no início das quatro estações do ano (outono - março; inverno - junho; primavera setembro; verão - dezembro), proporcionando assim uma análise do incremento médio anual e periódico por estação. $\mathrm{O}$ incremento foi avaliado com auxílio de paquímetro digital, com precisão milimétrica, obtendo-se a média dos incrementos de cada espécie por estação.

A avaliação da existência de agrupamentos na vegetação arbórea foi realizada pelo método Twinspan (Two-Way Indicator Species Analysis), o qual verifica a ocorrência de padrões na distribuição de espécies, associadas às condições ambientais a campo (HILL, 1979).

$\mathrm{Na}$ análise dos dados, foi utilizada uma matriz de 99x39, sendo 99 subparcelas (linhas) e 39 espécies (colunas). A utilização de 99 subparcelas deve-se ao fato de uma destas não apresentar indivíduos com CAP $\geq 30 \mathrm{~cm}$. A matriz teve como variáveis o número de indivíduos de cada uma das 39 espécies amostradas.

Seguindo a metodologia descrita por Nascimento (2000), Araújo (2002) e Narvaes (2004), as espécies observadas como raras (nesse estudo aquelas com menos de cinco indivíduos) foram descartadas da análise. Segundo Gauch (1982) as espécies com poucos indivíduos amostrados não influenciam na formação dos agrupamentos.

$\mathrm{Na}$ análise, os pontos de corte estipulados para as pseudoespécies foram $0,2,5,10$ e 20, o que consiste em representar todas as espécies de acordo com a proporção de indivíduos que ocorre em cada parcela. Posteriormente, as amostras e são agrupadas conforme sua similaridade.

$\mathrm{Na}$ definição dos agrupamentos, além da análise multivariada, foram consideradas as características observadas a campo como declividade, umidade do terreno e grau de alteração da parcela observada. Conforme Kent e Coker (1992), o número de subdivisões depende do tamanho e característica do conjunto 
de dados, associado aos fatores ambientais, considerando que não necessariamente todas as divisões são definidas como um grupo, mas aquelas que podem ser verificadas in loco e explicadas.

Finalmente, buscando descrever a vegetação em termos de sua dinâmica, foi verificado o incremento periódico e incremento anual para a floresta antes da análise de agrupamento e após a formação dos grupos. $\mathrm{O}$ incremento foi obtido pela diferença entre medidas consecutivas, considerando a medida do tempo 2 subtraída pela medida do tempo 1 .

\section{RESULTADOS E DISCUSSÃO}

\section{Caracterização da floresta}

Na vegetação amostrada na FLONA de São Francisco de Paula, considerando todos os indivíduos com CAP $\geq 30 \mathrm{~cm}$, observaram-se 64 espécies, pertencentes a 47 gêneros e 28 famílias botânicas, além de 9 espécies de cipós e 67 indivíduos mortos (Tabela 1).

TABELA 1: Composição florística, incremento médio anual $(\mathrm{cm})$ em diâmetro e incremento periódico $(\mathrm{cm})$ por estação do ano das espécies componentes da Floresta Ombrófila Mista da FLONA de São Francisco de Paula, RS, Brasil.

TABLE 1: Floristic composition and annual average increment $(\mathrm{cm})$ in diameter and periodic increment (cm) per season of the component species of Mixed Rainy Forest of FLONA in São Francisco de Paula, RS, Brazil.

\begin{tabular}{|c|c|c|c|c|c|}
\hline FAMÍLIA / Nome Científico & IPO & IPI & IPP & IPV & IMA \\
\hline $\begin{array}{l}\text { ANACARDIACEAE } \\
\text { Lithraea brasiliensis Marchand }\end{array}$ & 0,72 & 0,67 & 0,42 & 0,50 & 2,31 \\
\hline $\begin{array}{l}\text { ANNONACEAE } \\
\text { Rollinia rugulosa Schltdl. }\end{array}$ & 0,13 & 0,13 & 1,13 & 0,13 & 1,52 \\
\hline $\begin{array}{l}\text { AQUIFOLIACEAE } \\
\text { Ilex brevicuspis Reissek } \\
\text { Ilex dumosa Reissek } \\
\text { Ilex paraguariensis A. St.-Hil. }\end{array}$ & $\begin{array}{l}2,07 \\
1,65 \\
1,50\end{array}$ & $\begin{array}{l}1,56 \\
1,27 \\
1,30\end{array}$ & $\begin{array}{l}2,16 \\
3,08 \\
1,87\end{array}$ & $\begin{array}{l}4,64 \\
4,12 \\
2,88\end{array}$ & $\begin{array}{r}10,43 \\
10,12 \\
7,55\end{array}$ \\
\hline $\begin{array}{l}\text { ARAUCARIACEAE } \\
\text { Araucaria angustifolia (Bertol.) Kuntze }\end{array}$ & 1,14 & 1,07 & 1,51 & 1,57 & 5,29 \\
\hline $\begin{array}{l}\text { ASTERACEAE } \\
\text { Dasyphyllum spinescens (Less.) Cabrera } \\
\text { Dasyphyllum tomentosum (Spreng.) Cabrera } \\
\text { Vernonia discolor (Spreng.) Less. }\end{array}$ & $\begin{array}{l}1,10 \\
1,75 \\
2,40\end{array}$ & $\begin{array}{l}0,81 \\
1,50 \\
2,13\end{array}$ & $\begin{array}{l}1,19 \\
0,50 \\
2,13\end{array}$ & $\begin{array}{l}2,06 \\
2,25 \\
3,72\end{array}$ & $\begin{array}{r}5,16 \\
6,00 \\
10,38 \\
\end{array}$ \\
\hline $\begin{array}{l}\text { CELASTRACEAE } \\
\text { Maytenus evonymoides Reissek }\end{array}$ & 0,50 & 0,55 & 0,59 & 1,77 & 3,41 \\
\hline $\begin{array}{l}\text { CUNONIACEAE } \\
\text { Lamanonia ternata Vell. }\end{array}$ & 1,87 & 1,13 & 1,24 & 2,81 & 7,05 \\
\hline $\begin{array}{l}\text { DICKSONIACEAE } \\
\text { Dicksonia sellowiana Hook. }\end{array}$ & 1,06 & 1,18 & 1,14 & 5,25 & 8,63 \\
\hline $\begin{array}{l}\text { EUPHORBIACEAE } \\
\text { Sapium glandulatum (Vell.) Pax. } \\
\text { Sebastiania brasiliensis Spreng. } \\
\text { Sebastiania commersoniania (Baill.) L. B. Sm. et Downs }\end{array}$ & $\begin{array}{l}1,29 \\
1,16 \\
1,38\end{array}$ & $\begin{array}{l}0,77 \\
1,02 \\
1,12\end{array}$ & $\begin{array}{l}1,57 \\
0,92 \\
1,55\end{array}$ & $\begin{array}{l}2,38 \\
1,27 \\
1,83\end{array}$ & $\begin{array}{l}6,01 \\
4,37 \\
5,88\end{array}$ \\
\hline $\begin{array}{l}\text { FABACEAE } \\
\text { Inga vera Willd. } \\
\text { Lonchocarpus nitidus (Vogel) Benth. }\end{array}$ & $\begin{array}{l}1,12 \\
0,96\end{array}$ & $\begin{array}{l}0,73 \\
0,96\end{array}$ & $\begin{array}{l}0,85 \\
0,92\end{array}$ & $\begin{array}{l}1,25 \\
1,83\end{array}$ & $\begin{array}{l}3,95 \\
4,67\end{array}$ \\
\hline $\begin{array}{l}\text { FLACOURTIACEAE } \\
\text { Casearia decandra Jacq. } \\
\text { Casearia obliqua Spreng. } \\
\text { Xylosma pseudosalzmannii Sleumer }\end{array}$ & $\begin{array}{l}1,39 \\
1,48 \\
1,00\end{array}$ & $\begin{array}{l}1,17 \\
1,41 \\
1,08\end{array}$ & $\begin{array}{l}1,77 \\
1,41 \\
1,67\end{array}$ & $\begin{array}{l}2,54 \\
2,79 \\
1,08\end{array}$ & $\begin{array}{l}6,87 \\
7,09 \\
4,83 \\
\end{array}$ \\
\hline
\end{tabular}


TABELA 1: Continuação ...

TABLE 1: Continued ...

\begin{tabular}{|c|c|c|c|c|c|}
\hline FAMÍLIA / Nome Científico & IPO & IPI & IPP & IPV & IMA \\
\hline \multicolumn{6}{|l|}{ ICACINACEAE } \\
\hline Citronella gongonha (Mart.) Howard & 1,25 & 1,50 & 1,50 & 2,00 & 6,25 \\
\hline \multicolumn{6}{|l|}{ LAURACEAE } \\
\hline Cinnamomum aтоenum (Nees) Kosterm. & 0,25 & 0,25 & 0,25 & 0,25 & 1,00 \\
\hline Cinnamomum glaziovii (Mez) Kosterm. & 3,13 & 1,86 & 4,34 & 5,19 & 14,52 \\
\hline Cryptocarya aschersoniana Mez & 1,29 & 1,34 & 1,62 & 2,13 & 6,38 \\
\hline Nectandra megapotamica (Spreng.) Nez & 2,71 & 2,19 & 3,91 & 4,80 & 13,61 \\
\hline Ocotea indecora (Schott) Mez & 1,00 & 1,10 & 1,65 & 1,45 & 5,20 \\
\hline Ocotea puberula (A. Rich.) Nees & 2,19 & 2,83 & 2,76 & 3,16 & 10,94 \\
\hline Ocotea pulchella Mart. & 1,35 & 1,28 & 2,38 & 2,45 & 7,46 \\
\hline \multicolumn{6}{|l|}{ MELIACEAE } \\
\hline Cedrela fissilis Vell. & 3,00 & 2,25 & 2,50 & 9,50 & 17,25 \\
\hline \multicolumn{6}{|l|}{ MYRSINACEAE } \\
\hline Myrsine umbellata Mart. & 1,41 & 0,99 & 1,58 & 1,24 & 5,22 \\
\hline \multicolumn{6}{|l|}{ MYRTACEAE } \\
\hline Acca sellowiana (O. Berg) Burret & 0,25 & 0,25 & 0,25 & 0,25 & 1,00 \\
\hline \multicolumn{6}{|l|}{ MYRTACEAE } \\
\hline Blepharocalyx salicifolius (Kunth) O. Berg & 1,25 & 1,22 & 1,50 & 2,73 & 6,70 \\
\hline Calyptranthes concinna DC. & 0,66 & 0,61 & 0,86 & 1,39 & 3,52 \\
\hline Campomanesia rhombea O. Berg & 1,11 & 0,97 & 0,77 & 1,66 & 4,51 \\
\hline Campomanesia xanthocarpa O. Berg & 1,53 & 1,23 & 1,30 & 3,22 & 7,28 \\
\hline Eugenia involucrata DC. & 1,12 & 1,12 & 2,25 & 1,87 & 6,36 \\
\hline Eugenia psidiiflora O. Berg & 0,93 & 0,81 & 0,64 & 1,22 & 3,60 \\
\hline Eugemia uruguayensis Cambess. & 0,97 & 0,81 & 0,88 & 1,22 & 3,88 \\
\hline Myrceugenia cucullata D. Legrand & 1,38 & 1,15 & 1,30 & 1,63 & 5,46 \\
\hline Myrceugenia miersiana (Gardner) D. Legrand et Kausel & 0,50 & 0,50 & 0,33 & 0,25 & 1,58 \\
\hline Myrceugenia myrcioides (Cambess.) O. Berg & 0,50 & 0,50 & 0,50 & 0,50 & 2,00 \\
\hline Myrcia oligantha O. Berg & 0,56 & 0,66 & 0,56 & 0,40 & 2,18 \\
\hline Myrcianthes gigantea (D. Legrand) D. Legrand & 1,38 & 1,25 & 1,81 & 1,19 & 5,63 \\
\hline Myrcianthes pungens (O. Berg) D. Legrand & 0,50 & 0,25 & 0,25 & 0,50 & 1,50 \\
\hline Myrciaria floribunda (West ex Willd.) O. Berg & 0,92 & 0,83 & 1,58 & 1,63 & 4,96 \\
\hline Myrrhinium atropurpureum Schott & 0,67 & 1,00 & 0,67 & 0,58 & 2,92 \\
\hline Siphoneugena reitzii D. Legrand & 0,98 & 0,78 & 1,10 & 1,48 & 4,34 \\
\hline \multicolumn{6}{|l|}{ PHYTOLACCACEAE } \\
\hline Seguieria aculeata L. & 1,08 & 0,83 & 0,33 & 0,92 & 3,16 \\
\hline \multicolumn{6}{|l|}{ PODOCARPACEAE } \\
\hline Podocarpus lambertii Klotzsch ex Endl. & 0,93 & 0,85 & 1,29 & 1,57 & 4,64 \\
\hline \multicolumn{6}{|l|}{ PROTEACEAE } \\
\hline Roupala brasiliensis Klotzsch & 1,04 & 1,07 & 1,79 & 2,57 & 6,47 \\
\hline \multicolumn{6}{|l|}{ RHAMNACEAE } \\
\hline Scutia buxifolia Reissek & 1,09 & 0,66 & 0,62 & 0,68 & 3,05 \\
\hline \multicolumn{6}{|l|}{ ROSACEAE } \\
\hline Prunus myrtifolia (L.) Urb. & 2,36 & 1,65 & 2,94 & 4,46 & 11,41 \\
\hline \multicolumn{6}{|l|}{ RUTACEAE } \\
\hline Policarpus pennatifolius Lem. & 0,75 & 1,25 & 0,50 & 0,25 & 2,75 \\
\hline Zanthoxylum petiolare A. St.-Hil. et Tul. & 3,25 & 2,80 & 4,00 & 4,75 & 14,80 \\
\hline Zanthoxylum rhoifolium L. & 1,92 & 1,83 & 1,75 & 2,42 & 7,92 \\
\hline
\end{tabular}


TABELA 1: Continuação ...

TABLE 1: Continued ...

\begin{tabular}{lrrrrrr}
\hline FAMÍLIA / Nome Científico & IPO & IPI & IPP & IPV & IMA \\
\hline SAPINDACEAE & & & & & \\
Cupania vernalis Cambess. & 5,83 & 1,75 & 5,33 & 5,42 & 18,33 \\
Matayba elaeagnoides Radlk. & 1,63 & 2,02 & 2,57 & 5,02 & 11,24 \\
\hline SOLANACEAE & & & & & \\
Solanum mauritianum Scop. & 1,00 & 1,00 & 5,00 & 4,75 & 11,75 \\
Solanum sanctae-catharinae Dunal & 0,75 & 1,25 & 0,50 & 0,25 & 2,75 \\
\hline SYMPLOCACEAE & & & & & \\
Symplocos tetrandra (Mart.) Miq. & 0,75 & 0,75 & 2,25 & 4,00 & 7,75 \\
Symplocos uniflora (Pohl) Benth. & 0,87 & 0,62 & 1,00 & 0,37 & 2,86 \\
\hline THEACEAE & & & & & \\
Gordonia acutifolia (Wawra) H. Keng & 2,08 & 1,50 & 2,17 & 5,33 & 11,08 \\
\hline TILIACEAE & & & & & \\
Luehea divaricata Mart. et Zucc. & 1,88 & 1,71 & 1,81 & 4,69 & 10,09 \\
\hline ULMACEAE & & & & & \\
Celtis iguanaea (Jacq.) Sargent & 0,75 & 0,75 & 0,75 & 0,75 & 3,00 \\
\hline Cipós & 0,83 & 0,67 & 0,71 & 1,50 & 3,71 \\
\hline Incremento Médio & 1,42 & 1,17 & 1,61 & 2,42 & 6,62 \\
\hline
\end{tabular}

Em que: IPO = incremento periódico no outono; IPI = incremento periódico no inverno; IPP = incremento periódico na primavera; IPV = incremento periódico no verão; IMA = incremento médio anual.

As famílias Myrtaceae, com 11 gêneros e 17 espécies, e Lauraceae, com quatro gêneros e sete espécies, foram as mais representativas da floresta. Esse predomínio não é peculiaridade somente da Floresta Ombrófila Mista, sendo evidenciado em outras florestas de diversas regiões fitogeográficas do Rio Grande do Sul (LONGHI, 1997; RIO GRANDE DO SUL 2002; JARENKOW, 1985; VACCARO, 1997).

$\mathrm{O}$ índice de de Shannon foi de 3,53 indicando considerável diversidade florística. Comparativamente, Longhi (1997) estudando a Floresta Ombrófila Mista em diferentes bacias hidrográficas, constatou valores inferiores e superiores a esse, indicando que a diversidade florística é representativa da tipologia florestal estudada.

Os parâmetros dendrométricos das árvores amostradas na FLONA indicam valores médios de diâmetro, altura total estimada e altura comercial de $21,45 \mathrm{~cm}, 13,72 \mathrm{~m}$ e $8,21 \mathrm{~m}$ respectivamente. Os valores médios por hectare em relação ao número de árvores, área basal e volume comercial foram estimados em 885 árvores, $45,05 \mathrm{~m}^{2}$ e $326,92 \mathrm{~m}^{3} /$ ha respectivamente.

Os valores de densidade, área basal e volume médio por hectare nesse estudo foram superiores

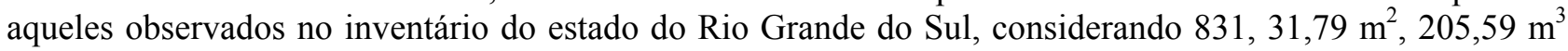
respectivamente (RIO GRANDE DO SUL, 2002). Entretanto, no mesmo estudo citado anteriormente, os valores máximos observados foram $1530,61,18 \mathrm{~m}^{2}$ e $440,36 \mathrm{~m}^{3}$, respectivamente, para densidade, área basal e volume.

Longhi (1997) observou área basal mínima de 20,66 $\mathrm{m}^{2} \mathrm{ha}^{-1}$ e máxima de $26,80 \mathrm{~m}^{2}$ ha ${ }^{-1}$ na região de Passo Fundo, RS. Enquanto Formento et al. (2004) observou $28,21 \mathrm{~m}^{3} \mathrm{ha}^{-1}$, na mesma tipologia florestal, no estado de Santa Catarina.

Nesse contexto, quando se analisa a floresta de forma geral é possível inferir que o ecossistema estudado se encontra em sítio de boa qualidade, possivelmente, em estágio avançado de sucessão.

Dentre as espécies avaliadas, as que apresentaram maior número de indivíduos foram: Araucaria angustifolia, Cryptocarya aschersoniana, Blepharocalyx salicifolius, Casearia decandra, Eugenia psidiiflora, Sebastiania brasiliensis, Ilex brevicuspis, Ilex paraguariensis, Sebastiania commersoniana, Sapium glandulatum, Nectandra megapotamica, Ocotea puchella e Eugenia uruguayensis. As demais espécies apresentaram menos de vinte indivíduos $\mathrm{ha}^{-1}$.

As espécies que apresentaram maior incremento foram Cupania vernalis, Cedrela fissilis, 
Zanthoxylum petiolare, Cinnamomum glaziovii, Nectandra megapotamica, Solanum mauritianum, Prunus myrtifolia, Matayba elaeagnoides, Gordonia acutifolia e Ocotea puberula, considerando todos os indivíduos amostrados (Tabela 1). Entre essas espécies, uma é pioneira, sete são secundárias iniciais e duas são secundárias tardias (VACCARO, 2002; MOSCOVICH, 2006). Conforme Ferreti (2002), as espécies comuns em ecossistemas recentemente alterados apresentam crescimento rápido apesar de, geralmente, apresentarem menor longevidade. Observa-se que somente $N$. megapotamica predominou na vegetação em termos de densidade (24 indivíduos $\mathrm{ha}^{-1}$ ), além disso, a sua característica de secundária inicial (MOSCOVICH, 2006), demonstra que as florestas apesar de estarem em estágio avançado de sucessão, provavelmente, mantêm as espécies iniciais em destaque em razão da abertura natural de clareiras, o que garante a dinâmica no ecossistema.

$\mathrm{Na}$ analise conjunta de todas as espécies, é possível descrever que a Floresta Ombrófila Mista da FLONA apresentou um incremento médio anual de $6,62 \mathrm{~cm}$, e avaliando-se o crescimento nas estações do ano, contatou-se que o maior incremento médio ocorreu no verão $(2,42 \mathrm{~cm})$, seguido da primavera $(1,61 \mathrm{~cm})$, outono $(1,42 \mathrm{~cm})$ e inverno $(1,17 \mathrm{~cm})$.

O verão ameno da região de estudo, conforme Taiz e Zeiger (2004), proporciona menor perda de água pelas folhas, permitindo a adequada absorção pelas raízes e translocação de minerais e, conseqüentemente, aumentando a produtividade do ecossistema. Em contrapartida, o inverno mais rigoroso reduz o crescimento nesta estação, considerando que a temperatura na qual o crescimento se processa mais rapidamente em regiões subtropicais e tropicais está entre 15 e $30{ }^{\circ} \mathrm{C}$ (LARCHER, 2000).

Conforme Leite \& Klein (1990), durante o inverno apesar de ocorrer precipitação intensa e regular, o frio restringe o desenvolvimento de muitas espécies.

\section{Análise de Agrupamento}

A Análise de Cluster pelo método de Twinspan definiu três grupos florísticos distintos entre as subparcelas avaliadas (Figura 1). Na formação dos grupos as espécies indicadoras, apresentam preferência ecológica para ocorrer em determinado local (HILL et al., 1975).

O autovalor de 0,5166 foi expressivo para definir a primeira divisão, mostrando a similaridade florística entre dois grupos de subparcelas, conforme pode ser observado na Figura 1. A maioria das espécies não foi exclusiva, porém ocorreram com maior abundância em um dos grupos de acordo com suas adaptação às condições ecológicas, assim sendo preferências a um determinado grupo. A Araucaria angustifolia foi a espécie indicadora do grupo 1, ocorrendo em 27 subparcelas. Nesse mesmo grupo, as pseudoespécies Ara ang 1 e 2 (Araucaria angustifolia em dois pontos de corte) e Luehea divaricata (Lue div 1) ocorreram em mais parcelas pertencentes ao grupo 1 do que nas parcelas do outro lado da divisão. As espécies preferenciais influenciam na formação do agrupamento numa primeira divisão; posteriormente, as espécies indicadoras definem o grupo de forma mais conclusiva. Os resultados da divisão, associados à tipologia da floresta e as observações a campo, indicam que esse grupo representa característica de ambiente clímax na Floresta Ombrófila Mista.

O outro grande grupo formado na primeira divisão apresentou Cryptocarya aschersoniana e Sebastiania brasiliensis como espécies indicadoras; e Cryptocarya aschersoniana, Eugenia psidiiflora, Eugenia uruguayensis, Nectandra megapotamica, Ocotea pulchella, Sapium glandulatum, Sebastiania brasiliensis e Sebastiania commersoniana como espécies preferenciais (Figura 1). Da mesma forma, pelas características das espécies, constitui o grupo das formações secundárias da floresta de áreas mais abertas e com solos mais úmidos. Esse mesmo grupo foi dividido novamente, validado pelo autovalor 0,4466, grando os grupos 2 e 3 (Figura 1).

O grupo 2 foi diferenciado por representar locais com maior umidade, sendo indicado pela presença de Sebastiania commersoniana (Seb com 1 em 14 subparcelas), Ocotea pulchella (Oco pul 1, em 13), Eugenia uruguayensis (Eug uru 1, em 11), Eugenia psidiiflora (Eug psi 1, em 13). Enquanto as espécies preferenciais foram Eugenia psidiiflora (Eug psi 1 e 2), Eugenia uruguayensis (Eug uru 1), Myrcia oligantha (Myr oli 1), Myrciaria floribunda (Myr flor 1), Ocotea pulchella (Oco pul 1), Roupala brasiliensis (Rou bra 1), Sebastiania commersoniana (Seb com 1 e 2) e Siphoneugena reitzii (Sip rei 1). 


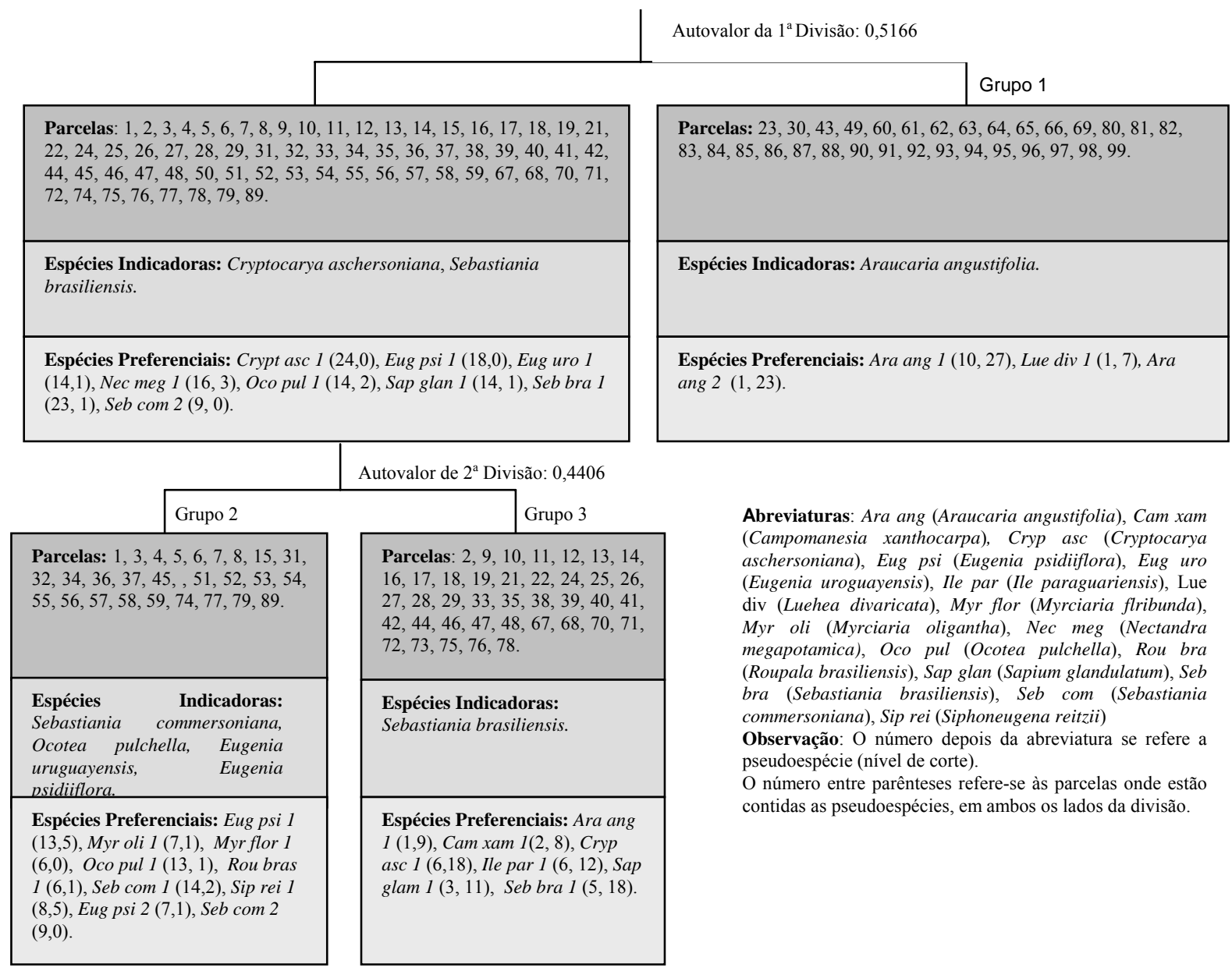

FIGURA 1: Classificação das unidades amostrais em três grupos ecológicos caracterizando as espécies componentes da Floresta Ombrófila Mista, FLONA de São Francisco de Paula, RS, Brasil.

FIGURE 1: Classification of the stands in three ecological groups characterizing the component species of Mixed Rainy Forest, FLONA in São Francisco de Paula, RS, Brazil.

O grupo 3 teve como espécie indicadora Sebastiania brasiliensis (Seb bra 1), presente em 18 subparcelas) e como espécies preferenciais Araucaria angustifolia (Ara ang 1), Campomanesia xanthocarpa (Can xan 1), Cryptocarya aschersoniana (Cry asc 1), Ilex paraguariensis (Ile par 1), Sapium glandulatum (Sap glan 1) e Sebastiania brasiliensis (Seb bra 1), sendo caracterizado pela presença de espécies tipicamente secundárias.

Os agrupamentos formados geraram novas divisões, porém, pelas observações de campo e pelos objetivos desejados no estudo, considerou-se a diferenciação de três grupos florísticos distintos, que demonstraram claramente as diferenças em relação à estrutura da área. Assim, de acordo com as características sucessionais das espécies indicadoras e preferenciais, os grupos foram denominados de grupo 1 - floresta primária; grupo 2 - floresta de locais úmidos; grupo 3 - floresta secundária.

\section{Caracterização dos grupos florísticos obtidos}

No grupo 1 (floresta primária) foram encontradas 38 espécies arbóreas pertencentes a 32 gêneros e 21 famílias botânicas, enquanto que no grupo 2 (floresta de locais úmidos) foram encontradas 38 espécies pertencentes a trinta gêneros e 19 famílias, no grupo 3 (floresta secundária) 47 espécies pertencentes a 36 gêneros e 21 famílias, entre os indivíduos com $C A P \geq 30,0 \mathrm{~cm}$, além de cipós (Tabelas 2, 3 e 4).

Em todos os grupos, as famílias Myrtaceae e Lauraceae foram as mais representativas. No grupo 1 
Myrtaceae, com cinco gêneros e cinco espécies, e Lauraceae, com quatro gêneros e cinco espécies; no grupo 2 Myrtaceae, com dez gêneros e 13 espécies, e Lauraceae, com três gêneros e quatro espécies; e no grupo 3 Myrtaceae, com dez gêneros e 14 espécies, e Lauraceae, com quatro gêneros e sete espécies. A predominância dessas famílias já foi observada em outros estudos no Rio Grande do Sul (JARENKOW, 1985; LONGHI, 1997; VACCARO, 1997; RIO GRANDE DO SUL, 2002; NARVAES, 2004).

Observa-se que o grupo 3 (Tabela 4) apresentou maior riqueza florística, por ser o grupo de formação secundária já em adiantado estágio de desenvolvimento, representado por espécies pioneiras associadas às espécies de estágio sucessional mais avançado. Entre as espécies pioneiras predominantes foram Casearia decandra, Dasyphyllum tomentosum, Lamanonia ternata, Myrsine sp., Myrsine umbellata e Sapium glandulatum (REITZ, KLEIN e REIS 1983; VACCARO, 1997).

No grupo 1, representado pela floresta primária (Tabela 2), predominaram as espécies Araucaria angustifolia, Casearia decandra, Ilex paraguariensis, Ilex brevicuspis, Luehea divaricata, Ilex dumosa e Blepharocalyx salicifolius. A única espécie exclusiva foi Lonchocarpus nitidus.

TABELA 2: Composição florística, incremento médio anual $(\mathrm{cm})$ em diâmetro e incremento periódico $(\mathrm{cm})$ por estação do ano das espécies componentes do grupo 1, na Floresta Ombrófila Mista da FLONA de São Francisco de Paula, RS, Brasil.

TABLE 2: Floristic composition and annual average increment $(\mathrm{cm})$ in diameter and periodic increment $(\mathrm{cm})$ per season of the component species of group 1, in Mixed Rainy Forest of FLONA, São Francisco de Paula, RS, Brazil.

\begin{tabular}{l|c|c|c|c|c|c}
\hline \multicolumn{1}{c}{ Espécies } & IPO & IPI & IPP & IPV & IMA & N \\
\hline Araucaria angustifolia & 1,11 & 1,04 & 1,55 & 1,61 & 5,31 & 86 \\
Blepharocalyx salicifolius & 1,12 & 1,16 & 1,34 & 2,45 & 6,07 & 10 \\
Campomanesia xanthocarpa & 1,75 & 1,25 & 1,00 & 1,00 & 5,00 & 1 \\
Casearia decandra & 1,55 & 1,44 & 2,00 & 3,28 & 8,26 & 15 \\
Casearia obliqua & 1,35 & 1,29 & 1,54 & 2,41 & 6,59 & 4 \\
Cedrela fissilis & 3,00 & 2,25 & 2,50 & 9,50 & 17,25 & 1 \\
Cinnamomum glaziovii & 3,50 & 2,06 & 4,88 & 5,67 & 16,10 & 6 \\
Citronella gongonha & 1,25 & 1,50 & 1,50 & 2,00 & 6,25 & 2 \\
Cryptocarya moschata & 2,05 & 1,55 & 1,55 & 2,05 & 7,20 & 1 \\
Cupania vernalis & 4,50 & 1,25 & 3,25 & 3,75 & 12,75 & 1 \\
Dasyphyllum spinescens & 1,41 & 1,06 & 1,06 & 2,56 & 6,10 & 4 \\
Eugenia uruguayensis & 1,88 & 1,25 & 1,13 & 1,75 & 6,00 & 2 \\
Ilex brevicuspis & 1,79 & 1,75 & 2,12 & 3,96 & 9,61 & 12 \\
Ilex dumosa & 1,63 & 1,18 & 1,83 & 3,25 & 7,88 & 10 \\
Ilex paraguariensis & 1,16 & 1,08 & 1,76 & 2,51 & 6,51 & 15 \\
Inga vera & 1,54 & 0,75 & 1,04 & 1,93 & 5,25 & 7 \\
Lamanonia ternata & 2,25 & 1,43 & 1,00 & 2,46 & 7,14 & 7 \\
Lonchocarpus nitidus & 0,96 & 0,96 & 0,92 & 1,83 & 4,67 & 6 \\
Luehea divaricata & 1,91 & 1,73 & 1,82 & 4,55 & 10,00 & 11 \\
Matayba elaeagnoides & 1,96 & 1,83 & 2,67 & 5,21 & 11,67 & 6 \\
Maytenus evonymoides & 1,67 & 1,92 & 2,00 & 7,67 & 13,25 & 3 \\
Myrceugenia cucullata & 1,38 & 1,19 & 1,41 & 1,88 & 5,84 & 8 \\
Myrcianthes gigantea & 2,00 & 1,50 & 0,50 & 1,25 & 5,25 & 1 \\
Myrsine umbellata & 1,63 & 0,70 & 0,70 & 0,83 & 3,85 & 2 \\
Nectandra megapotamica & 3,08 & 3,00 & 5,08 & 5,75 & 16,92 & 3 \\
Ocotea puberula & 0,30 & 0,30 & 0,30 & 2,40 & 3,30 & 1 \\
Ocotea pulchella & 1,00 & 1,00 & 1,13 & 1,19 & 4,31 & 4 \\
Pilocarpus pennatifolius & 0,75 & 1,25 & 0,50 & 0,25 & 2,75 & 1 \\
\hline
\end{tabular}


TABELA 2: Continuação ...

TABLE 2: Continued ...

\begin{tabular}{l|c|c|c|c|c|c}
\hline \multicolumn{1}{c|}{ Espécies } & IPO & IPI & IPP & IPV & IMA & N \\
\hline Prunus myrtifolia & 1,38 & 1,50 & 1,88 & 3,25 & 8,00 & 2 \\
Rollinia rugulosa & 0,00 & 0,00 & 2,00 & 0,00 & 2,00 & 1 \\
Sapium glandulatum & 1,25 & 1,25 & 2,00 & 3,00 & 7,50 & 1 \\
Scutia buxifolia & 0,50 & 0,50 & 0,50 & 0,50 & 2,00 & 1 \\
Sebastiania brasiliensis & 1,00 & 1,50 & 0,50 & 0,50 & 3,50 & 1 \\
Sebastiania commersoniana & 1,50 & 1,50 & 0,50 & 0,50 & 4,00 & 1 \\
Solanum mauritianum & 1,00 & 1,00 & 5,00 & 4,75 & 11,75 & 1 \\
Xylosma pseudosalzmanii & 1,17 & 1,17 & 1,00 & 1,33 & 4,67 & 2 \\
Zanthoxylum petiolare & 3,25 & 2,80 & 4,00 & 4,75 & 14,80 & 1 \\
Zanthoxylum rhoifolium & 0,88 & 1,00 & 0,88 & 2,00 & 4,75 & 2 \\
Valores Médios & 1,61 & 1,34 & 1,74 & 2,78 & 7,47 & \\
\hline
\end{tabular}

Em que: IPO = incremento periódico no outono; IPI = incremento periódico no inverno; IPP = incremento periódico na primavera; IPV = incremento periódico no verão; IMA = incremento médio anual.

No grupo 2, da floresta de locais úmidos (Tabela 3), as espécies Eugenia psidiiflora, Sebastiania commersoniana, Cryptocarya aschersoniana, Myrcia oligantha, Ilex brevicuspis, Blepharocalyx salicifolius e Eugenia uruguayensis foram as predominantes, tendo Myrceugenia miersiana e Myrcia oligantha, como exclusivas.

TABELA 3: Composição florística, incremento médio anual $(\mathrm{cm})$ em diâmetro e incremento periódico $(\mathrm{cm})$ por estação do ano das espécies componentes do grupo 2, na Floresta Ombrófila Mista da FLONA de São Francisco de Paula, RS, Brasil.

TABLE 3: Floristic composition and annual average increment $(\mathrm{cm})$ in diameter and periodic increment (cm) per season of the component species of group 2, in Mixed Rainy Forest of FLONA, São Francisco de Paula, RS, Brazil.

\begin{tabular}{l|c|c|c|c|c|c}
\hline \multicolumn{1}{c}{ Espécies } & IPO & IPI & IPP & IPV & IMA & N \\
\hline Blepharocalyx salicifolius & 1,40 & 1,22 & 1,52 & 2,66 & 6,79 & 10 \\
Calyptranthes concinna & 0,83 & 0,55 & 0,88 & 1,50 & 3,77 & 3 \\
Campomanesia rhombea & 1,88 & 1,58 & 0,83 & 1,13 & 5,40 & 2 \\
Campomanesia xanthocarpa & 1,65 & 1,30 & 1,15 & 3,25 & 7,35 & 5 \\
Casearia decandra & 1,29 & 1,29 & 2,00 & 1,83 & 6,42 & 6 \\
Celtis iguanaea & 0,75 & 0,75 & 0,75 & 0,75 & 3,00 & 1 \\
Cipós & 0,25 & 0,25 & 0,25 & 2,25 & 3,00 & 1 \\
Cryptocarya aschersoniana & 1,37 & 1,26 & 1,11 & 1,76 & 5,50 & 21 \\
Dicksonia sellowiana & 1,13 & 1,00 & 0,88 & 8,50 & 11,50 & 4 \\
Eugenia involucrata & 1,00 & 0,75 & 3,75 & 1,50 & 7,00 & 1 \\
Eugenia psidiiflora & 0,88 & 0,84 & 0,65 & 1,13 & 3,51 & 24 \\
Eugenia uruguayensis & 0,65 & 0,60 & 0,65 & 0,75 & 2,63 & 10 \\
Gordonia acutifolia & 1,25 & 1,25 & 2,00 & 7,50 & 12,00 & 1 \\
Ilex brevicuspis & 1,64 & 1,41 & 1,89 & 4,70 & 9,64 & 11 \\
Ilex dumosa & 1,75 & 1,58 & 7,25 & 7,00 & 17,58 & 3 \\
Ilex paraguariensis & 1,63 & 2,38 & 1,25 & 3,13 & 8,38 & 4 \\
Lamanonia ternata & 1,63 & 0,63 & 0,69 & 1,31 & 4,25 & 4 \\
Luehea divaricata & 1,50 & 1,50 & 1,75 & 6,25 & 11,00 & 1 \\
Matayba elaeagnoides & 2,00 & 1,00 & 1,50 & 3,50 & 8,00 & 1 \\
Myrceugenia cucullata & 1,75 & 1,08 & 1,33 & 1,42 & 5,58 & 3 \\
Myrceugenia miersiana & 0,50 & 0,50 & 0,33 & 0,25 & 1,58 & 6 \\
\hline
\end{tabular}


TABELA 3: Continuação ...

TABLE 3: Continued ...

\begin{tabular}{l|c|c|c|c|c|c}
\hline \multicolumn{1}{c|}{ Espécies } & IPO & IPI & IPP & IPV & IMA & N \\
\hline Myrcia oligantha & 0,56 & 0,66 & 0,56 & 0,40 & 2,18 & 13 \\
Myrcianthes gigantea & 1,13 & 1,25 & 2,75 & 1,00 & 6,13 & 2 \\
Myrciaria floribunda & 0,81 & 0,75 & 1,50 & 0,88 & 3,94 & 4 \\
Myrsine umbellata & 1,92 & 1,17 & 2,17 & 1,67 & 6,92 & 3 \\
Nectandra megapotamica & 2,69 & 2,02 & 2,04 & 3,64 & 10,39 & 6 \\
Ocotea indecora & 1,06 & 1,13 & 1,81 & 1,56 & 5,56 & 4 \\
Ocotea pulchella & 1,11 & 1,03 & 2,96 & 2,06 & 7,17 & 8 \\
Podocarpus lambertii & 0,50 & 0,50 & 0,75 & 0,75 & 2,50 & 1 \\
Prunus myrtifolia & 4,50 & 1,00 & 1,00 & 0,50 & 7,00 & 1 \\
Rollinia rugulosa & 0,25 & 0,25 & 0,25 & 0,25 & 1,00 & 1 \\
Roupala brasiliensis & 1,05 & 1,00 & 2,15 & 3,10 & 7,30 & 5 \\
Sapium glandulatum & 0,38 & 0,25 & 0,25 & 1,25 & 2,13 & 2 \\
Scutia buxifolia & 1,39 & 0,74 & 0,55 & 0,83 & 3,50 & 4 \\
Sebastiania brasiliensis & 1,20 & 1,11 & 1,22 & 0,84 & 4,37 & 8 \\
Sebastiania commersoniana & 1,46 & 1,06 & 1,64 & 1,89 & 6,04 & 22 \\
Seguieria aculeata & 1,08 & 0,83 & 0,33 & 0,92 & 3,17 & 3 \\
Siphoneugena reitzii & 1,06 & 0,72 & 1,22 & 1,38 & 4,38 & 8 \\
Vernonia discolor & 1,00 & 1,00 & 1,00 & 1,00 & 4,00 & 1 \\
\hline Valores Médios & 1,29 & 1,00 & 1,45 & 2,20 & 5,94 & - \\
\hline
\end{tabular}

Em que: IPO = incremento periódico no outono; IPI $=$ incremento periódico no inverno; IPP $=$ incremento periódico na primavera; IPV = incremento periódico no verão; IMA = incremento médio anual.

Já no grupo 3, floresta secundária (Tabela 4) houve predomínio de Cryptocarya aschersoniana, Eugenia psidiiflora, Sebastiania brasiliensis, Sapium glandulatum, Blepharocalyx salicifolius, Casearia decandra, Nectandra megapotamica e Ilex paraguariensis. A única espécie exclusiva do grupo foi Myrrhinium atropurpureum.

As árvores do grupo 1 apresentaram maiores dimensões, com valores médios para diâmetro, altura total e comercial de $23,34 \mathrm{~cm}, 15,44 \mathrm{~m}$ e $9,89 \mathrm{~m}$ respectivamente, enquanto para o grupo 2 esses parâmetros foram de 22,57 cm, 13,06 m e 7,34 m, e para o grupo 3 de 21,29 cm, 14,47 m e 7,93 m, respectivamente. Esses valores foram superiores às médias encontradas para Floresta Ombrófila Mista no inventário contínuo do estado do Rio Grande do Sul, onde foi descrito $19,42 \mathrm{~cm}, 11,65 \mathrm{~m}$ e 6,28 m respectivamente (RIO GRANDE DO SUL, 2002).

TABELA 4: Composição florística, incremento médio anual $(\mathrm{cm})$ em diâmetro e incremento periódico $(\mathrm{cm})$ por estação do ano das espécies componentes do grupo 3, na Floresta Ombrófila Mista da FLONA de São Francisco de Paula, RS, Brasil.

TABLE 4: Floristic composition and annual average increment $(\mathrm{cm})$ in diameter and periodic increment (cm) per season of the component species of group 3, in Mixed Rainy Forest of FLONA, São Francisco de Paula, RS, Brazil.

\begin{tabular}{lcccccc}
\hline \multicolumn{1}{c}{ Espécies } & IPO & IPI & IPP & IPV & IMA & N \\
\hline Acca sellowiana & 0,25 & 0,25 & 0,25 & 0,25 & 1,00 & 1 \\
Araucaria angustifolia & 1,36 & 1,32 & 1,27 & 1,27 & 5,23 & 11 \\
Blepharocalyx salicifolius & 1,26 & 1,27 & 1,61 & 3,02 & 7,16 & 21 \\
Calyptranthes concinna & 0,53 & 0,65 & 0,84 & 1,31 & 3,33 & 4 \\
Campomanesia rhombea & 0,97 & 0,86 & 0,81 & 1,93 & 4,57 & 11 \\
Campomanesia xanthocarpa & 1,44 & 1,19 & 1,42 & 3,44 & 7,50 & 9 \\
Casearia decandra & 1,28 & 0,91 & 1,51 & 2,15 & 5,86 & 19 \\
Cinnamomum amoenum & 0,25 & 0,25 & 0,25 & 0,25 & 1,00 & 1 \\
\hline
\end{tabular}


TABELA 4: Continuação ...

TABLE 4: Continued ...

\begin{tabular}{|c|c|c|c|c|c|c|}
\hline Espécies & IPO & IPI & IPP & IPV & IMA & $\mathrm{N}$ \\
\hline Cinnamomum glaziovii & 2,00 & 1,25 & 2,75 & 3,75 & 9,75 & 2 \\
\hline Cipós & 0,95 & 0,75 & 0,80 & 1,35 & 3,85 & 5 \\
\hline Cryptocarya aschersoniana & 1,22 & 1,40 & 2,03 & 2,42 & 7,08 & 26 \\
\hline Cupania vernalis & 6,50 & 2,00 & 6,38 & 6,25 & 21,13 & 2 \\
\hline Dasyphyllum tomentosum & 0,50 & 0,50 & 1,00 & 0,25 & 2,25 & 1 \\
\hline Dicksonia sellowiana & 1,00 & 1,35 & 1,41 & 2,00 & 5,76 & 4 \\
\hline Eugenia involucrata & 1,25 & 1,50 & 0,75 & 2,25 & 5,75 & 1 \\
\hline Eugenia psidiiflora & 1,13 & 0,79 & 0,65 & 1,55 & 4,12 & 10 \\
\hline Eugenia uruguayensis & 1,13 & 0,96 & 1,09 & 1,62 & 4,81 & 9 \\
\hline Gordonia acutifólia & 2,50 & 1,63 & 2,25 & 4,25 & 10,63 & 2 \\
\hline Ilex brevicuspis & 3,00 & 1,47 & 2,56 & 5,54 & 12,57 & 9 \\
\hline Ilex paraguariensis & 1,83 & 1,23 & 2,16 & 3,20 & 8,43 & 14 \\
\hline Inga vera & 0,63 & 0,71 & 0,63 & 0,46 & 2,42 & 6 \\
\hline Lamanonia ternata & 1,58 & 1,11 & 1,88 & 4,21 & 8,78 & 6 \\
\hline Lithraea brasiliensis & 0,72 & 0,67 & 0,42 & 0,50 & 2,30 & 3 \\
\hline Matayba elaeagnoides & 1,50 & 2,38 & 3,00 & 5,92 & 12,79 & 6 \\
\hline Myrceugenia cucullata & 0,83 & 1,08 & 0,83 & 1,00 & 3,73 & 2 \\
\hline Myrcianthes gigantea & 1,25 & 1,00 & 1,25 & 1,50 & 5,00 & 1 \\
\hline Myrcianthes pungens & 0,50 & 0,25 & 0,25 & 0,50 & 1,50 & 1 \\
\hline Myrciaria floribunda & 1,13 & 1,00 & 1,75 & 3,13 & 7,00 & 2 \\
\hline Myrrhinium atropurpureum & 0,67 & 1,00 & 0,67 & 0,58 & 2,92 & 3 \\
\hline Myrsine sp. & 0,85 & 0,88 & 1,88 & 3,25 & 6,85 & 2 \\
\hline Myrsine umbellate & 0,90 & 0,82 & 0,98 & 0,82 & 3,52 & 3 \\
\hline Nectandra megapotamica & 2,65 & 2,10 & 4,42 & 5,07 & 14,23 & 15 \\
\hline Ocotea indecora & 0,75 & 1,00 & 1,00 & 1,00 & 3,75 & 1 \\
\hline Ocotea puberula & 2,50 & 3,25 & 3,17 & 3,29 & 12,21 & 6 \\
\hline Ocotea pulchella & 1,74 & 1,66 & 2,49 & 2,99 & 8,87 & 9 \\
\hline Podocarpus lambertii & 0,96 & 0,88 & 1,33 & 1,63 & 4,79 & 14 \\
\hline Prunus myrtifolia & 2,30 & 1,97 & 4,30 & 6,58 & 15,15 & 3 \\
\hline Roupala brasiliensis & 1,00 & 1,25 & 0,88 & 1,25 & 4,38 & 2 \\
\hline Sapium glandulatum & 1,36 & 0,77 & 1,40 & 2,13 & 5,65 & 22 \\
\hline Scutia buxifolia & 0,50 & 0,50 & 1,00 & 0,25 & 2,25 & 1 \\
\hline Sebastiania brasiliensis & 1,16 & 0,97 & 0,85 & 1,43 & 4,41 & 26 \\
\hline Sebastiania commersoniana & 1,15 & 1,32 & 1,57 & 1,79 & 5,83 & 6 \\
\hline Siphoneugena reitzii & 0,82 & 0,86 & 0,86 & 1,36 & 3,89 & 6 \\
\hline Solanum sanctae-catharinae & 0,75 & 1,25 & 1,00 & 1,00 & 4,00 & 1 \\
\hline Symplocos tetranda & 0,75 & 0,75 & 2,25 & 4,00 & 7,75 & 1 \\
\hline Symplocos uniflora & 0,88 & 0,63 & 1,00 & 0,38 & 2,88 & 2 \\
\hline Xylosma pseudosalzmannii & 1,25 & 1,25 & 2,50 & 1,50 & 6,50 & 1 \\
\hline Zanthoxylum rhoifolium & 4,00 & 3,50 & 3,50 & 3,25 & 14,25 & 1 \\
\hline Valor & 1,36 & 1,15 & 1,64 & 2,26 & 6,44 & - \\
\hline
\end{tabular}

Em que: IPO = incremento periódico no outono; IPI = incremento periódico no inverno; IPP = incremento periódico na primavera; IPV = incremento periódico no verão; IMA = incremento médio anual.

A floresta primária (Grupo 1) apresentou maiores valores de área basal $\left(51,58 \mathrm{~m}^{2} / \mathrm{ha}\right)$ e volume comercial $\left(450,95 \mathrm{~m}^{3} / \mathrm{ha}\right)$, explicado pela existência de indivíduos de grande porte, sobretudo de Araucaria angustifolia. Por outro lado, a floresta de locais úmidos apresentou elevado número de indivíduos por hectare (939,29 indivíduos), fato esse comum em ambientes com elevada saturação hídrica, conforme já descrito por Pagano e Durigan (2000).

Na floresta primária (Tabela 2), o incremento médio em diâmetro foi de 2,78 cm no verão, $1,74 \mathrm{~cm}$ 
na primavera, 1,61 cm no outono e $1,34 \mathrm{~cm}$ no inverno. Na floresta de locais úmidos (Tabela 3), o incremento foi de $2,20 \mathrm{~cm}$ no verão, $1,45 \mathrm{~cm}$ na primavera, $1,29 \mathrm{~cm}$ no outono e $1,00 \mathrm{~cm}$ no inverno. Na floresta secundária (Tabela 4), apresentou incremento médio no verão de $2,26 \mathrm{~cm}$, seguido da primavera de $1,64 \mathrm{~cm}$, outono de $1,36 \mathrm{~cm}$ e inverno de $1,15 \mathrm{~cm}$.

Os resultados indicaram que o crescimento das árvores está diretamente relacionado às estações climáticas anuais (períodos do ano), no estado do Rio Grande do Sul. Dessa forma, em todos os grupos, houve maior incremento médio em diâmetro no verão, seguido da primavera, outono e inverno.

No que se refere ao incremento médio anual em diâmetro, observou-se que a floresta primária (Tabela 2) apresentou maior valor $(7,47 \mathrm{~cm})$, possivelmente, por estar localizada no sítio de melhor qualidade e encontrar-se estabelecida. A floresta de locais úmidos (Tabela 3) apresentou crescimento médio anual em diâmetro de, aproximadamente, $5,94 \mathrm{~cm}$ e a floresta secundária (Tabela 4) de 6,44 cm.

O menor incremento da floresta de locais úmidos pode ser explicado pela umidade, limitante do crescimento de muitas espécies. Conforme Taiz e Zeiger (2004), a maioria das plantas necessita de oxigênio quando está envolvida em atividades metabólicas, com exceção de algumas espécies que apresentam adaptações à anoxia, o crescimento e a sobrevivência são bastante prejudicadas em ambientes com baixa concentração de Oxigênio, podendo reduzir a produtividade. Já a floresta secundária (grupo 3), apresentou incremento médio anual intermediário, provavelmente, em conseqüência da presença de um maior número de espécies competindo, por outro lado, foi, nesse ambiente, que ocorreu a espécie com maior incremento (Cupania vernalis). Neste contexto, Cupania vernalis foi a favorecida em relação às demais espécies.

$\mathrm{Na}$ análise dos ecótonos, observou-se que, na floresta clímax, o maior incremento ocorreu para Cedrella fissilis $(17,25 \mathrm{~cm})$, seguida por Nectandra megapotamica $(16,92 \mathrm{~cm})$, Cinnamomum glaziovii $(16,10 \mathrm{~cm})$, Zanthoxylum petiolare $(14,80 \mathrm{~cm})$ (Tabela 2); na floresta com locais úmidos, foram Ilex dumosa $(17,58 \mathrm{~cm})$, Gordonia acutifolia $(12,00 \mathrm{~cm})$, Dicksonia sellowiana $(11,50 \mathrm{~cm})$, Luehea divaricata $(11,00 \mathrm{~cm})$ (Tabela 3); e no grupo 3, associação secundária, de forma crescente as espécies que apresentaram maior incremento foram Cupania vernalis $(21,13 \mathrm{~cm})$, Prunus myrtifolia $(15,15 \mathrm{~cm})$, Nectandra megapotamica $(14,23 \mathrm{~cm})$, Matahyba elaeagnoides (12,79 cm) (Tabela 4).

O destaque de Nectandra megapotamica na análise geral da floresta foi corroborado por sua presença e incremento expressivo nos grupos 1 e 3. A Cupania vernalis por ser uma espécie secundária (MOSCOVICH, 2006) e de crescimento rápido foi fortemente favorecida no processo competitivo, do ecótono floresta secundária. Assim, ambas as espécies merecem destaque e, provavelmente, estarão presentes no processo evolutivo da floresta.

\section{CONCLUSÕES}

A análise geral da floresta sugere que esta apresenta característica clímax com processos naturais de mudança, o qual mantém as espécies pioneiras. Entretanto, a classificação pelo TWINSPAN indica a presença de agrupamentos (ecótonos) diferentes que devem ser manejados de forma independente;

No manejo na FLONA de São Francisco de Paula, deve-se considerar diferentes ecótonos representados por floresta clímax, secundária e de locais úmidos. Estes apresentam diferentes características na associação de espécies;

A análise geral da floresta indicou maior incremento no verão $(2,42 \mathrm{~cm})$ seguido por primavera $(1,61$ $\mathrm{cm})$, outono $(1,42)$ e inverno $(1,17)$. A mesma tendência foi observada nos ecótonos.

A análise e descrição geral de uma floresta poderá restringir decisões para planos de manejo, visto a existência de agrupamentos com associações de espécies e incrementos distintos;

De forma mais ampla, em programas silviculturais e de manejo florestal, recomenda-se dar ênfase para as espécies Araucaria angustifolia, Cedrela fissilis, Cinnamomum glaziovii, Cupania vernalis, Diksonia sellowiana, Ilex brevicuspis, Ilex paraguariensis, Luehea divaricata, Matayba elaeagnoides, Nectandra megapotamica, Ocotea pulchella, Prunus myrtifolia e Roupala brasiliensis por apresentarem maiores incrementos médios anuais em diâmetro e possuírem potencialidades econômicas, considerando suas preferências ecológicas. 


\section{REFERÊNCIAS BIBLIOGRÁFICAS}

ARAUJO, M. M. Vegetação e mecanismos de regeneração em fragmento de Floresta Estacional Decidual Ripária, Cachoeira do Sul, RS, Brasil. 2002. 153f. Tese (Doutorado em Engenharia Florestal) - Universidade Federal de Santa Maria, Santa Maria, 2002.

BOTOSSO, P. C.; TOMAZELLO FILHO, M. Aplicação de faixas dendrométricas na dendrocronologia: avaliação da taxa e do ritmo de crescimento do tronco de árvores tropicais e subtropicais. In: MAIA, N. B., MARTOS, H. L.; BARELLA, W. (Org.). Indicadores ambientais: conceitos e aplicações. São Paulo: EDUC/COMPED/INEP, 2001. p.145-171.

FELFILI, J. M.; SILVA Jr., M. C. da.; SEVILHA, A. C.; REZENDE, A. V.; NOGUEIRA, P. E.; WALTER, B. M. T.; SILVA, F. das C.; SALGADO, M. A. Fitossociologia da vegetação arbórea. In: FELFILI, J. M.; SILVA Jr., M. C. da. (Org.). Biogeografia do Bioma Cerrado: estudo fitofisionômico na Chapada do Espigão Mestre do São Francisco. Brasília: UnB, 2001. p.35-68.

FELFILI, J. M.; SEVILHA, A. C.; SILVA Jr., M. C. da. Comparação entre as unidades fisiogrãficasChapada Pratinha, Veadeiros e Espigão Mestre de São Francisco. In: FELFILI, J. M.; SILVA Jr., M. C. da (Org.). Biogeografia do Bioma Cerrado: estudo fitofisionômico na Chapada do Espigão Mestre do São Francisco. Brasília: UnB, 2001. p. 8094.

FERRETTI, A. R. Modelos de Plantio para a Restauração. In: GALVÃO, A. P. M.; MEDEIROS, A. C. de S. (Org.). A restauração da Mata Atlântica em áreas de sua primitiva ocorrência natural. Colombo: Embrapa Florestas. 2002, p. 35-43.

FORMENTO, S. SCHORN, L. A.; RAMOS, R. A. B. Dinâmica estrutural arbórea de uma Floresta Ombrófila Mista em Campo Belo do Sul, SC. Cerne, Lavras, v.10, n.2, p.196-212, 2004.

GAUCH, H. G. Multivariate analysis in community ecology. Cambridge: Cambridge University Press, 1982.180 p.

GAUTO, O. A. Análise da dinâmica e impactos da exploração sobre o estoque remanescente (por espécies e grupos de espécies similares) de uma Floresta Estacional Semidecidual em Misiones, Argentina. 1997. 133f. Dissertação (Mestrado em Engenharia Florestal) - Universidade Federal do Paraná, Curitiba, 1997.

HIGUSHI, N. et al. Uso de bandas metálicas e dendrômetros automáticos para a definição do padrão de crescimento individual das principais espécies arbóreas da floresta primária da região de Manaus, Amazonas, Brasil. In: Projeto Jacarandá FASE II: Pesquisas florestais na Amazônia Central. Manaus: INPE, 2003. p. 55-68.

HILL, M. O. TWINSPAN: a FORTRAN program for arranging multivariate data in an ordered two-way table by classification of the individuals and attributes. Ithaca, NY: Cornell University, 1979. 60p.

HILL, M. O.; BUNCE, R. G. H.; SHAW, M. W. Indicator species analysis, a divisive polythetic method of classification, and its application to a survey of native pinewoods in Scotland. The Journal of Ecology, Oxford, v. 63, n.2, p.597-613, 1975.

JARENKOW, J. A. Composição Florística e Estrutura da Mata com Araucária na Estação Ecológica de Aracuri, Esmeralda, Rio Grande do Sul. 1985. 86f. Dissertação (Mestrado em Ciências Biológicas) - Universidade Federal do Rio Grande do Sul, Porto Alegre, 1985.

KENT, M.; COKER, P. Vegetation description and analysis. London: Behaven Press, 1992. 363p.

LAMPRECHT, H. Silvicultura nos trópicos: Ecossistemas florestais e respectivas espécies arbóreas: possibilidades e métodos de aproveitamento sustentado. Eschborn: Instituto de Silvicultura da Universidade de Göttingen/GTZ, 1990. $343 \mathrm{p}$.

LARCHER, W. Ecofisiologia vegetal. São Carlos: Rima, 2000. 531p.

LEITE, P. F.; KLEIN, R. M. Vegetação. In: IBGE. Fundação Instituto Brasileiro de Geografia e Estatística. Geografia do Brasil: Região Sul. Rio de Janeiro: 1990, p.113- 150. v. 2.

LONGHI, S. J. Agrupamento e análise fitossociológica de comunidades florestais na sub-bacia hidrográfica do Rio Passo Fundo- RS. 1997. 198f. Tese (Doutorado em Engenharia Florestal) - Universidade Federal do Paraná, Curitiba, 1997.

Mc CUNE, B.; MEFFORD, M. J. PC-ORD. Multivariate Analysis of Ecological Data. Oregon, USA: 1997. 40p.

MORENO, J. A. Clima do Rio Grande do Sul. Porto Alegre: Secretaria da Agricultura, 1961. 42p.

MOSCOVICH, F. A. Dinâmica de crescimento de uma Floresta Ombrófila Mista em Nova Prata, RS. $2006.130 f$. Tese (Doutorado em Engenharia Florestal) - Universidade Federal de Santa Maria, Santa Maria, 2006. 
NASCIMENTO, A. R. T. Análise estrutural e padrões de distribuição espacial de uma amostra de Floresta Ombrófila Mista. 2000. 90f. Dissertação (Mestrado em Engenharia Florestal) - Universidade Federal de Santa Maria, Santa Maria, 2000.

NARVAES, I. S. da. Classificação e caracterização da regeneração natural em Floresta Ombrófila Mista na Floresta Nacional de São Francisco de Paula, RS. 2004. 114f. Dissertação (Mestrado em Engenharia Florestal) Universidade Federal de Santa Maria, Santa Maria, 2004.

NIMER, E. Clima. In: IBGE. Fundação Instituto Brasileiro de Geografia e Estatística. Geografia do Brasil: Região Sul. Rio de Janeiro: 1990. p.151-187.

PAGANO, S. N.; DURIGAN, G. Aspectos da ciclagem de nutrientes em matas ciliares do oesta do estado de São Paulo, Brasil. RODRIGUES, R. R.; LEITÃO FILHO, H. de F. (Org.). Matas ciliares: conservação e recuperação. São Paulo: Editora de Universidade de São Paulo : FAPESP, 2000. p.109-123.

REITZ, R.; KLEIN, R. M. Araucariáceas. In: REITZ, R. (Org.). Flora ilustrada catarinense. Itajaí: Herbário Barbosa Rodrigues, 1966. $63 \mathrm{p}$.

REITZ, R.; KLEIN, R. M.; REIS, A. Projeto Madeira do Rio Grande do Sul. Sellowia, Itajaí, n.34-35, p.1-525, 1983.

RIO GRANDE DO SUL. Governo do Estado. Secretaria Estadual do Meio Ambiente. Inventário florestal contínuo do Rio Grande do Sul. Porto Alegre: SEMA, 2002. 706p. (Relatório Final).

SANTOS, J. H. S.; FERREIRA, R. L. G.; SILVA, J. A. A. da; SOUZA, A. L. de; SANTOS, E. de S.; MEUNIER, I. M. J. Distinção de grupos ecológicos de espécies florestais por meio de técnicas multivariadas. Revista Árvore, Viçosa, v. 28, n.3, p 387-396, 2004.

SOUZA, A. L.; ARAUJO, P. A.; CAMPOS, C.C.; NETO, F. P. Dinâmica de crescimento em diâmetro de uma floresta primária sem interferência: uma análise pelo tempo de passagem entre classes diamétricas. Revista Árvore, Viçosa, v.17, n.2, p.129-145, 1993.

TAIZ, L.; ZEIGER, E. Fisiologia vegetal. 3.ed. Porto Alegre : Artmed, 2004. 719p.

VACCARO, S. Caracterização fitossociológica de três fases sucessionais de uma floresta estacional decidual, no município de Santa Tereza - RS. 1997. 92f. Dissertação (Mestrado em Engenharia Florestal) - Universidade Federal de Santa Maria, Santa Maria, 1997.

VACCARO, S. Crescimento de uma floresta estacional decidual, no município de Santa Tereza, RS, Brasil. 2002. 137f. Tese (Doutorado em Engenharia Florestal) - Universidade Federal de Santa Maria, Santa Maria, 2002. 